\title{
UNIVERSITY OF MIAMI RADIOCARBON DATES III
}

\section{K L ELDRIDGE, J J STIPP, and S J COHEN}

Department of Geology, University of Miami, Coral Gables, Florida

The following radiocarbon measurements made since our last date

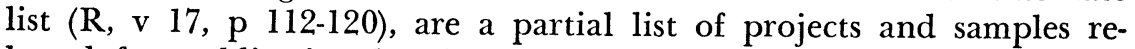
leased for publication by the submitters. The technique employed is liquid scintillation counting of wholly synthesized benzene as described by Noakes et al (1965) and discussed in R, v 16, p 402-408. Errors are reported as one standard deviation. No correction factors are applied.

\section{ACKNOWLEDGMENTS}

We are very grateful to D Evans, Dept of Biology for the supplemental use of his Packard Tri-Carb 2003 liquid scintillation spectrometer.

\section{SAMPLE DESCRIPTIONS}

\section{ARCHAEOLOGIC SAMPLES}

\section{A. United States}

\section{UM-205. Broward County charcoal}

Sample from $155 \mathrm{~cm}$ beneath surface, $1.6 \mathrm{~km} \mathrm{~N}$ of Hollywood Blvd, $.8 \mathrm{~km} \mathrm{~W}$ of State Rd \#7, Broward Co, Florida $\left(26^{\circ} 01^{\prime} 59^{\prime \prime} \mathrm{N}, 80^{\circ} 26^{\prime}\right.$ 09" W). Coll 1974 by W F Coleman; subm 1974 by F T Huna, MiamiWest India Arch Soc, Miami, Florida. Comment (FTH): dates habitation by early $S$ Florida Indians.

\section{GEOLOGIC SAMPLES}

\section{Shackelford Banks series}

\section{A. United States}

Two wood samples: SH-13 from $2.4 \mathrm{~km} \mathrm{~W}$ of Cape Lookout Lighthouse, off coast of North Carolina (34 $34^{\prime} 28^{\prime \prime} \mathrm{N}, 76^{\circ} 33^{\prime} 50^{\prime \prime} \mathrm{W}$ ); SH-1 from W end of Shackelford Banks, 46m SW of Mullet Pond, near

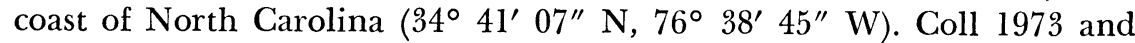
subm 1974 by K Susman, Duke Univ.

General Comment (KS): dates stratigraphic sequence for Shakelford Banks.

\section{UM-187. Shakelford SH-1}

$12,280 \pm 370$

From $14 \mathrm{~m}$ water.

$$
10,330 \mathrm{BC}
$$

\section{UM-188. Shakelford SH-13}

$\mathbf{2 4 , 5 3 5} \pm \mathbf{8 0 0}$

From 23m water.

\section{2,585 BC}

\section{Snapper Point series}

Mangrove peat from 4 cores, Snapper Point, Key Largo, Florida 
$\left(25^{\circ} 19^{\prime} 57^{\prime \prime} \mathrm{N}, 80^{\circ} 17^{\prime} 24^{\prime \prime} \mathrm{W}\right)$. Coll and subm 1974 by E R Rich, Dept Biol, Univ Miami.

General Comment: all peat samples were pretreated with $5 \%$ hot $\mathrm{NaOH}$, $10 \%$ hot $\mathrm{HCl}$, rinsed with deionized $\mathrm{H}_{2} \mathrm{O}$ and dried.

General Comment (ERR): dates used as relative indicators of current processes in stable, land-mangrove areas. Cores 1, 3, and 4 have similar decay and environmental histories. Core 5 is from an anaerobic, offshore deposit, indicating an earlier shoreline. Visible root hairs were handpicked by submitter.

UM-232. Core 1,10 to $20 \mathrm{~cm}$

Modern

Comment (ERR): questionable whether material at this interval was formed in situ.

\begin{tabular}{|c|c|c|}
\hline UM-233. & Core 1,20 to $30 \mathrm{~cm}$ & $\operatorname{AD} 1470$ \\
\hline UM-234. & Core 1,40 to $50 \mathrm{~cm}$ & $\begin{array}{r}775 \pm 60 \\
\text { AD } 1175\end{array}$ \\
\hline UM-235. & Core 1,60 to $70 \mathrm{~cm}$ & $\begin{array}{r}1130 \\
\text { AD } 820\end{array}$ \\
\hline UM-236. & Core 3,10 to $20 \mathrm{~cm}$ & $\begin{array}{r}270 \pm 85 \\
\text { AD } 1680\end{array}$ \\
\hline UM-237. & Core 3,40 to $50 \mathrm{~cm}$ & $\underset{A D 840}{1110} \pm 105$ \\
\hline UM-238. & Core 3,70 to $80 \mathrm{~cm}$ & $\begin{array}{r}1450 \pm 145 \\
\text { AD 500 }\end{array}$ \\
\hline UM-239. & Core 4,10 to $20 \mathrm{~cm}$ & $\begin{array}{r}190 \pm 95 \\
\text { AD } 1760\end{array}$ \\
\hline UM-240. & Core 4,30 to $40 \mathrm{~cm}$ & $\begin{array}{r}505 \\
\text { AD } 1445\end{array}$ \\
\hline UM-241. & Core 4,60 to $70 \mathrm{~cm}$ & $\begin{aligned} 1465 & \pm 75 \\
\operatorname{AD} 485 & \end{aligned}$ \\
\hline UM-242. & Core 5,0 to $10 \mathrm{~cm}$ & $\begin{array}{r}1350 \pm 80 \\
\text { AD } 600\end{array}$ \\
\hline
\end{tabular}

Comment (ERR): sample from shallow bottom community containing live marine algae and other organisms.

\section{Lake Okeechobee series}

Lake samples studied to determine environmental effect of backpumping on marsh areas; to reconstruct sedimentary environment of lake; to date onset of peat accumulation and end of marl deposition. Coll 1973 and subm 1974 by P J Gleason, C \& S F Flood Control Dist, Palm Beach, Florida. 
UM-190. Lake Okeechobee, LO-1

$12,050 \pm 210$

Marl from Lake Okeechobee bottom sediments, $\mathrm{S}$ lake Okeechobee, Florida $\left(26^{\circ} 52^{\prime} \mathrm{N}, 80^{\circ} 45^{\prime} \mathrm{W}\right)$.

UM-191. Lake Okeechobee, Core $11: 0-2$

$860 \pm 120$

Muck from 0 to $5 \mathrm{~cm}$, Kreamer I., Lake Okeechobee, Florida $\left(26^{\circ}\right.$ $\left.46^{\prime} \mathrm{N}, 80^{\circ} 44^{\prime} \mathrm{W}\right)$. Comment (PJG): sample contained high ash content.

\section{UM-192. Lake Okeechobee, Core 11:103-107}

Peat from 262 to $272 \mathrm{~cm}$, same as UM-191. Comment (PJG): age is minimum for onset of peat deposition.

\section{$\begin{array}{ll} & 6470 \pm 120 \\ \text { UM-193. Lake Okeechobee, Core 11:108-109 } & \begin{array}{l}4520 \mathrm{BC}\end{array}\end{array}$}

Calcitic marl from 274 to $276 \mathrm{~cm}$, same as UM-191. Comment (PJG): date represents end of marl deposition.

UM-194. Lake Okeechobee, Core 12:18-20

$3055 \pm 80$

1105 BC

Sandy peat from 46 to $51 \mathrm{~cm}$, NE conservation Area 3, Broward Co, Florida $\left(26^{\circ} 15^{\prime} \mathrm{N}, 80^{\circ} 30^{\prime} \mathrm{W}\right)$.

UM-195. Lake Okeechobee, Core $13: 24-27$

$1445 \pm 75$

AD 505

Sandy peat from 61 to $69 \mathrm{~cm}, \mathrm{~N}$ conservation Area 2B, Broward Co, Florida $\left(26^{\circ} 12^{\prime} \mathrm{N}, 80^{\circ} 24^{\prime} \mathrm{W}\right)$.

UM-196. Lake Okeechobee, Core 14:9-11

$3460 \pm 80$

1510 BC

Sandy peat from 23 to $28 \mathrm{~cm}$, S conservation Area 2B, Broward Co, Florida $\left(26^{\circ} 08^{\prime} \mathrm{N}, 80^{\circ} 22^{\prime} \mathrm{W}\right)$.

\section{DeSoto Canyon series}

Two cores of silty clay, rich in calcareous faunas, from continental slope, DeSoto Canyon, Gulf of Mexico. Core GS-7102-5 from NW of canyon $\left(29^{\circ} 17^{\prime} \mathrm{N}, 87^{\circ} 15^{\prime} \mathrm{W}\right)$. Core GS-7102-9 from SE of canyon $\left(29^{\circ} 00^{\prime} \mathrm{N}, 87^{\circ} 00^{\prime} \mathrm{W}\right)$. Coll 1973 by $\mathrm{S}$ Gartner; subm 1973 by $\mathrm{C}$ Emiliani, RSMAS, Miami, Florida.

General Comment (CE): Core GS-7102-5 contains some detrital carbonate establishing maximum ${ }^{14} \mathrm{C}$ values for samples. Dates are part of study of paleoclimatology of Quaternary sediments from NE Gulf of Mexico. Because of upwelling, climatic record is preserved in greater detail than typical pelagic oozes.

UM-61. GS-7102-5, 32 to $69 \mathrm{~cm}$

UM-60. GS-7102-5, 132 to $169 \mathrm{~cm}$
$12,925 \pm 200$ 10,975 BC

$18,390 \pm 205$ 16,440 BC 
UM-59. GS-7102-5, 235 to $265 \mathrm{~cm}$

UM-58. GS-7102-5, 385 to $415 \mathrm{~cm}$

UM-57. GS-7102-5, 485 to $515 \mathrm{~cm}$

UM-257. GS-7102-9, 35 to $65 \mathrm{~cm}$

UM-258. GS-7102-9, 65 to $100 \mathrm{~cm}$

UM-259. GS-7102-9, 100 to $120 \mathrm{~cm}$

UM-260. GS-7102-9, 120 to $140 \mathrm{~cm}$

UM-261. GS-7102-9, 183 to $200 \mathrm{~cm}$

UM-262. GS-7102-9, 200 to $220 \mathrm{~cm}$

UM-263. GS-7102-9, 230 to $250 \mathrm{~cm}$

UM-264. GS-7102-9, 250 to $270 \mathrm{~cm}$

UM-265. GS-7102-9, 290 to $310 \mathrm{~cm}$

UM-311. GS-7102-9, 350 to $370 \mathrm{~cm}$

UM-312. GS-7102-9, 370 to $390 \mathrm{~cm}$
$23,135 \pm 410$

21,185 BC

30,145

$+1930$

$-2550$

28,195 вC

$>42,500$

$5735 \pm 75$ 3785 BC

$8640 \pm 190$ $6690 \mathrm{BC}$

$10,865 \pm 145$ 8915 BC

$12,220 \pm 140$ $10,270 \mathrm{BC}$

$16,310 \pm 200$ $14,360 \mathrm{BC}$

$17,280 \pm 195$ 15,330 вС

$17,885 \pm 170$ 15,935 BC

$17,885^{+500}$

15,935 BC

$20,625+610$

18,675 BC

$21,640+390$

$19,690 \mathrm{BC}$

$25,040+545$

23,090 BC

$23,260+590$

21,310 BC 
UM-313. GS-7102-9, 490 to $510 \mathrm{~cm}$

UM-314. GS-7102-9, 510 to $530 \mathrm{~cm}$
23,085 вC

$27,560+860$

25,610 вC

\section{Edisto Beach series}

Shell from 3 areas of Edisto I, Charleston Co, South Carolina: Edingsville samples from $.8 \mathrm{~km}$ offshore $\left(32^{\circ} 31^{\prime} \mathrm{N}, 80^{\circ} 16^{\prime} \mathrm{W}\right)$; Bay Point Beach Ridge samples (32 $28^{\prime} \mathrm{N}, 80^{\circ} 20^{\prime} \mathrm{W}$ ); Botany Bay samples from intertidal zone $\left(32^{\circ} 33^{\prime} \mathrm{N}, 80^{\circ} 12^{\prime} \mathrm{W}\right)$. Mercenaria valves from Privateer Creek, Seabrook I, Charleston Co, South Carolina $\left(32^{\circ} 34^{\prime} \mathrm{N}\right.$, $80^{\circ} 19^{\prime} \mathrm{W}$ ). Coll and subm 1974 by F W Stapor, Jr, South Carolina Wildlife \& Marine Resources Dept.

UM-206. Edingsville C-1

Mercenaria valves from recrystallized calcarenite. Calcarenite is substrate for vermetid reef.

UM-207. Edingsville C-2

Mercenaria valves. Comment (FWS): UM-206 and -207 date formation of vermetid substrate.

UM-225. Edingsville R-1

Vermetid-serpulid tubes.

UM-226. Edingsville R-2

Vermetid-serpulid tubes.

UM-227. Edingsville R-3

Vermetid-serpulid tubes.

UM-251. Edingsville R-4

Vermetid-serpulid tubes.

UM-252. Edingsville R-5

Vermetid-serpulid tubes.

UM-255. Edingsville R-5b
AD 1390

$560 \pm 100$

$575 \pm 75$ AD 1375

AD 1150

$3990 \pm 90$

2040 BC

AD 1270

$680 \pm 80$

$835 \pm 75$

AD 1115

Outer chalky fraction of UM-252. Comment: less radiogenic than apparently unaltered inner fraction. 
UM-208. Bay Point A-1

Mercenaria shells from 1 to $2 \mathrm{~m}$ beneath surface. Sample from oldest area of beach ridge-plain.

UM-229. Bay Point A-1b

$1540 \pm 75$

Outer chalky fraction of UM-208. Comment: less radiogenic than apparently unaltered inner fraction.

UM-209. Bay Point A-2

$1710 \pm 85$

Mercenaria valves from 1 to $2 \mathrm{~m}$ beneath surface. Sample from oldest area of beach-ridge plain.

UM-230. Bay Point A-2b

$3020 \pm 70$

Outer chalky fraction of UM-209. Comment: less radiogenic than apparently unaltered inner fraction.

UM-243. Bay Point A-3

$2635 \pm 80$

$685 \mathrm{BC}$

Mercenaria shells from 1 to $2 \mathrm{~m}$ beneath surface. Sample from oldest area of beach-ridge plain.

UM-253. Bay Point A-3b

$2530 \pm 75$

Outer chalky fraction of UM-243. Comment: more radiogenic than apparently unaltered inner fraction.

UM-210. Bay Point B-1

$1490 \pm 70$

Mercenaria valves from 1 to $2 \mathrm{~m}$ beneath surface. Sample from 2 nd oldest area of beach-ridge plain.

UM-211. Bay Point B-2

$1390 \pm 70$

Mercenaria valves from 1 to $2 \mathrm{~m}$ beneath surface. Sample from 2 nd oldest area of beach-ridge plain.

UM-212. Bay Point B-3

$2525 \pm 90$

Mercenaria shells from 1 to $2 \mathrm{~m}$ beneath surface. Sample from 2 nd oldest area of beach-ridge plain.

UM-213. Bay Point C-1

$1550 \pm 70$ AD 400

Mercenaria shells from 2 to $3 \mathrm{~m}$ beneath surface. Sample from 2nd youngest area of beach-ridge plain.

UM-214. Bay Point C-2

$1685 \pm 100$ AD 265

Mercenaria shells from 2 to $3 \mathrm{~m}$ beneath surface. Sample from $2 \mathrm{nd}$ youngest area of beach-ridge plain. 


\section{UM-231. Bay Point C-2b}

$1915 \pm 105$

Outer chalky fraction of UM-214. Comment: less radiogenic than apparently unaltered inner fraction.

UM-215. Bay Point C-3

$$
31,915^{+1370}
$$

Mercenaria shells from 2 to $3 \mathrm{~m}$ beneath surface. Sample from 2 nd youngest area of beach-ridge plain. Comment: date anomalously older than expected.

\section{UM-216. Bay Point D-1}

Mercenaria shells from 1 to $2 \mathrm{~m}$ beneath surface. Sample from youngest area of beach-ridge plain.

\section{UM-217. Bay Point D-2}

$330 \pm 65$

Mercenaria shells from 1 to $2 \mathrm{~m}$ beneath surface. Sample from youngest area of beach-ridge plain.

\section{UM-220. Botany Bay}

$9145 \pm 160$

Large pelecypod and gastropod shells.

\section{UM-221. Botany Bay}

$7195 \mathrm{BC}$

Small pelecypod and gastropod shells.

\section{UM-218. Botany Bay}

$3600 \pm 85$

$1650 \mathrm{BC}$

Small pelecypod and gastropod shells.

\section{UM-219. Botany Bay}

$4830 \pm 90$

2880 BC

Small pelecypod and gastropod shells.

\section{UM-247. Botany Bay}

$8915 \pm 170$

6965 BC

Anadara valves.

UM-248. Botany Bay

$2475 \pm 70$

525 BC

Anadara valves.

\section{UM-254. Botany Bay}

$3480 \pm 70$

$1530 \mathrm{BC}$

Outer chalky fraction of UM-248. Comment: more radiogenic than apparently unaltered inner fraction.

UM-249. Botany Bay

$1200 \pm 75$

Dinocardium valves. 
UM-250. Botany Bay

$3030 \pm 110$

Dinocardium valves.

UM-222. Seabrook Island Beach Ridge 1

$5280 \pm 110$

3330 BC

26,300

UM-223. Seabrook Island Beach Ridge 2

24,350 BC

UM-224. Seabrook Island Beach Ridge 3

$1250 \pm 70$ AD 700

UM-244. Seabrook Island Beach Ridge 4

$1365 \pm 75$

AD 585

$1170 \pm 60$

UM-245. Seabrook Island Beach Ridge 5

AD 780

$31,920+1370$

$-1650$

UM-246. Seabrook Island Beach Ridge $6 \quad 29,970$ BC

B. Territoire Français des Afars et des Issas

\section{UM-228. Afar Depression}

$6565 \pm 235$

$4615 \mathrm{BC}$

Shell from Afar Depression, Territoire Français des Afars et des Issas $\left(11^{\circ} 35^{\prime} \mathrm{N}, 42^{\circ} 28^{\prime} \mathrm{E}\right)$. Coll 1972 and subm 1974 by C G A Harrison and E Bonatti, RSMAS, Miami, Florida. Comment (EB): dates desiccation of this section of Afar Depression. Area is center of active extension and spreading, genetically connected to Sheba Ridge in Gulf of Aden. Hyaloclastites coll indicate an underwater eruption.

REFERENCES

Noakes, J E, Kim, S M, and Stipp, J J, 1965, Chemical and counting advances in liquid scintillation age dating: 6 th internatl ${ }^{14} \mathrm{C}$ and ${ }^{3} \mathrm{H}$ dating conf Proc, Pullman, Washington, June 7-11, 1965, p 68-92. 